\title{
Editorial
}

\section{Geometry of Banach Spaces, Operator Theory, and Their Applications}

\author{
Genqi Xu, ${ }^{1}$ Ji Gao, ${ }^{2}$ Peide Liu, ${ }^{3}$ and Satit Saejung ${ }^{4}$ \\ ${ }^{1}$ Department of Mathematics, Tianjin University, Tianjin 300072, China \\ ${ }^{2}$ Department of Mathematics, Community College of Philadelphia, Philadelphia, PA 19130-3991, USA \\ ${ }^{3}$ College of Mathematics and Statistics, Wuhan University, Wuhan 430072, China \\ ${ }^{4}$ Department of Mathematics, Khon Kaen University, Khon Kaen 40002, Thailand
}

Correspondence should be addressed to Genqi Xu; gqxu@tju.edu.cn

Received 2 January 2014; Accepted 2 January 2014; Published 18 February 2014

Copyright (C) 2014 Genqi Xu et al. This is an open access article distributed under the Creative Commons Attribution License, which permits unrestricted use, distribution, and reproduction in any medium, provided the original work is properly cited.

In recent years, with developments of computer, high technique, and life science, more and more requirements were proposed to functional analysis. There were a large number of research articles published in the relative area and many major discoveries have been obtained. At the present time the ideas, terminology, and methods of functional analysis have penetrated deeply, not only into natural sciences, but also into applied disciplines. Many real world problems were analyzed and solved by using the ideas and results of function analysis. To fit the requirements, we edit this special issue.

The special issue represented the recent development in geometric structure of Banach spaces, Bergman spaces, and Minkowski spaces, random normed modules on function space, linear and nonlinear operator theory, and the applications of modern analysis in related areas of mathematics, as well as other disciplines, such as economics, finance and risk management, dynamic systems, the natural and life sciences, medicine, physics, and other real world problems.

The special issue consisted of twenty research articles contributed by participants of the 4th International Conference on Modern Analysis and Its Applications which was held at the Tianjin University, China, from August 1 to 4, 2013. With over 240 participants from different countries, the conference proved highly successful in bringing together experts and researchers and provided an opportunity to evaluate and disseminate new ideas and methods in modern analysis.

Each paper has been peer-reviewed and the editors thank the referees for their time and efforts to help ensure a high standard for this special issue.
We hope that readers interested in function spaces and operator theory will find in this special issue not only new information about development of functional analysis, but also important questions be resolved and directions for further study. At same time, we hope that in the near future, we can see new results to be published based on this special issue.

\section{Acknowledgment}

The auditors acknowledge Chinese Mathematical Society, National Nature Science Foundation of China, and the Department of Mathematics of Tianjin University, China. Without their support the conference would not have been possible. Thanks are also to editorial office of the Journal of Function Spaces and Applications.

Genqi Xu Ji Gao

Peide Liu

Satit Saejung 


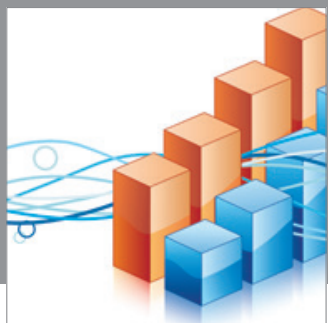

Advances in

Operations Research

mansans

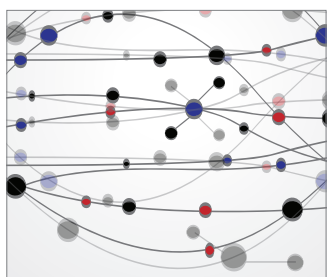

The Scientific World Journal
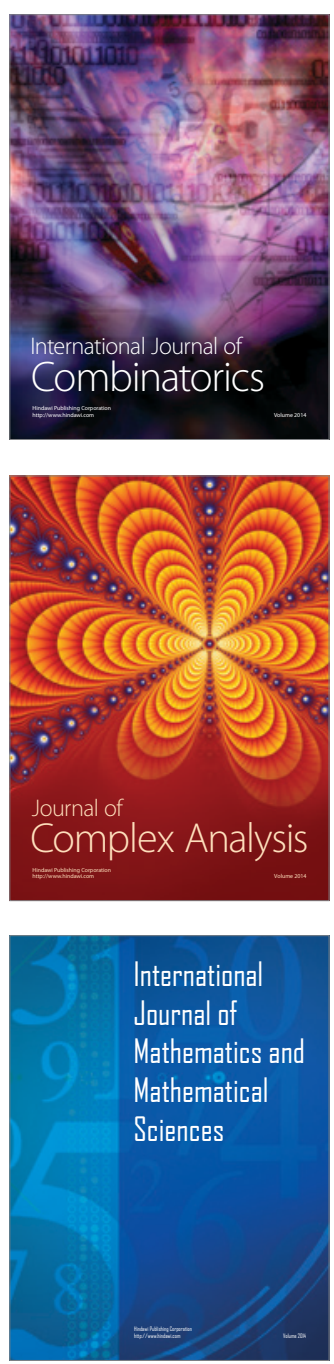
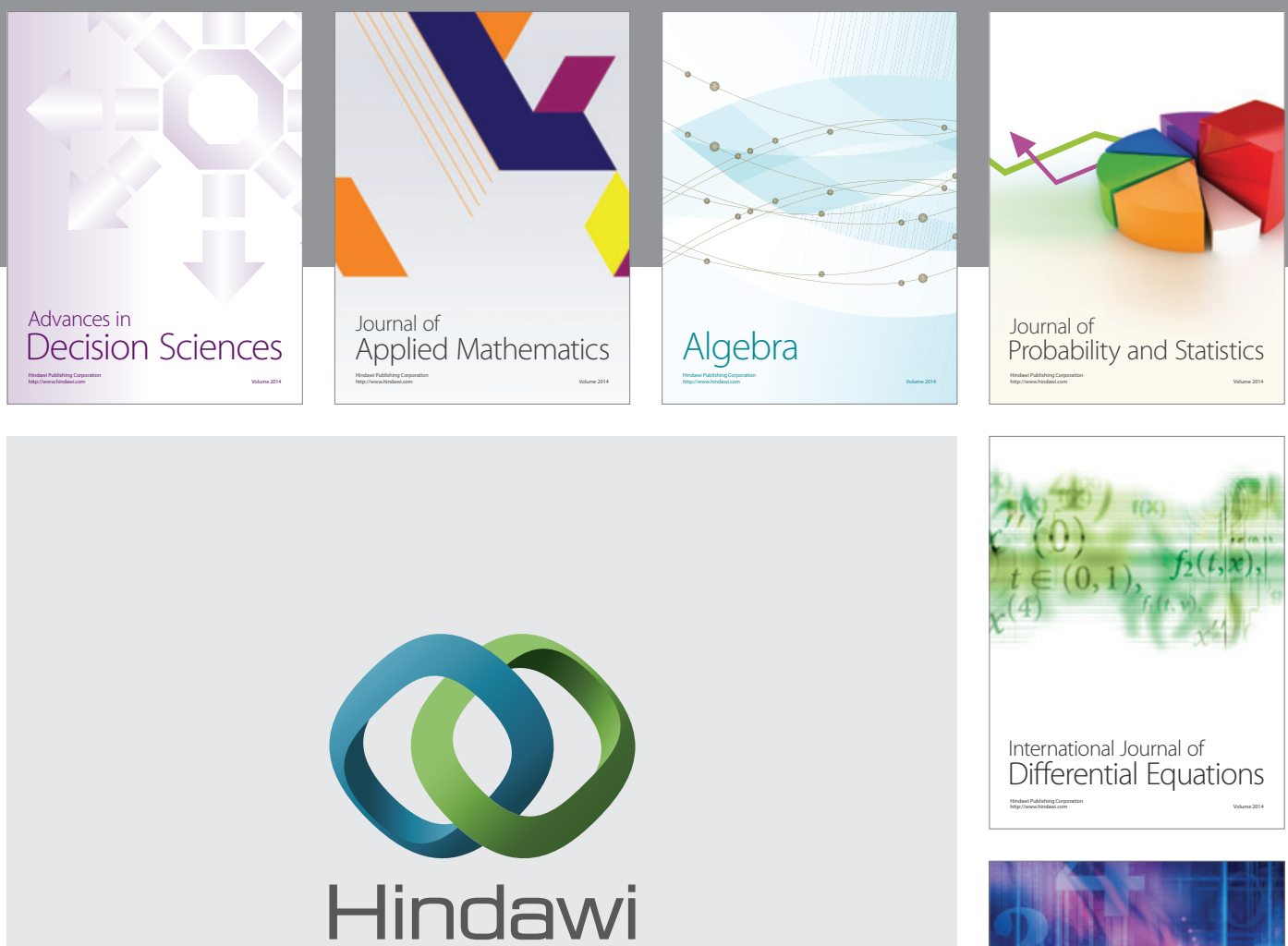

Submit your manuscripts at http://www.hindawi.com
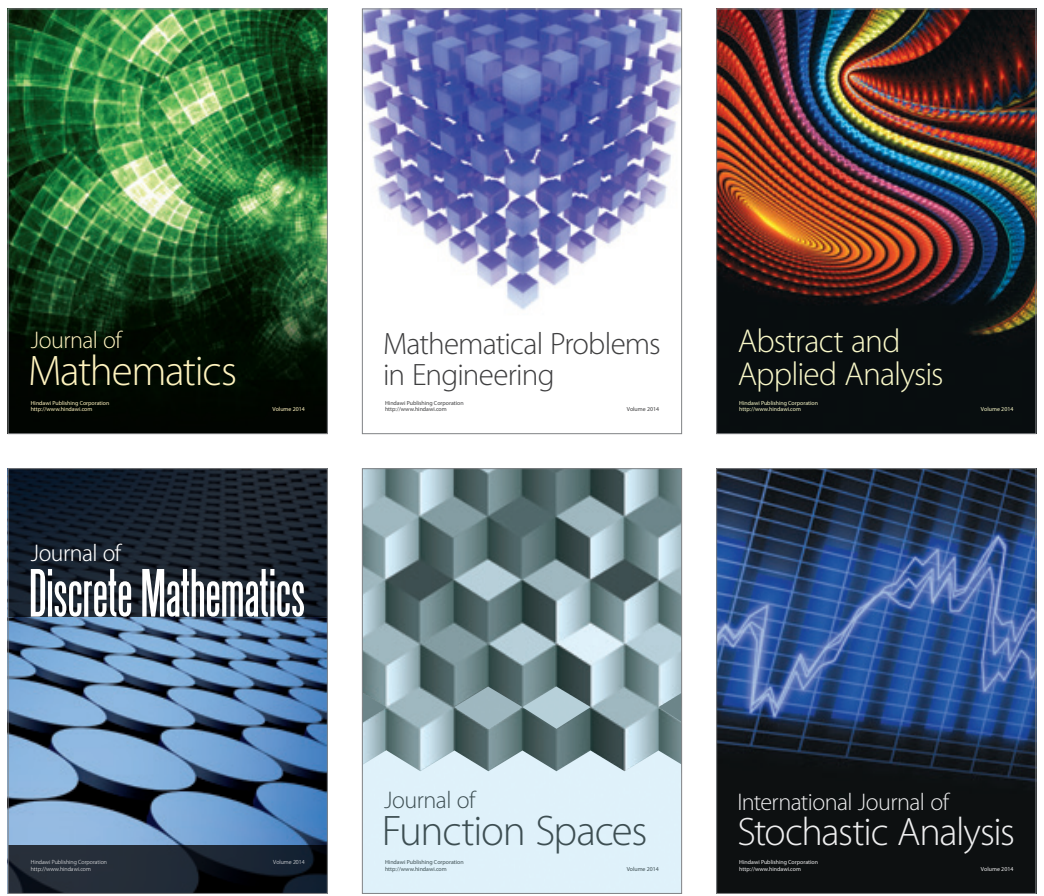

Journal of

Function Spaces

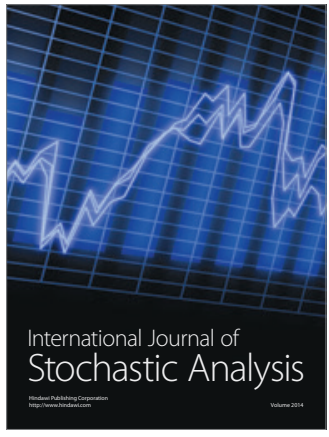

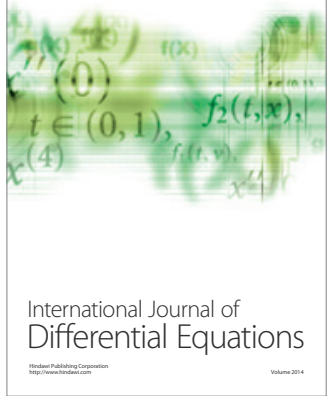
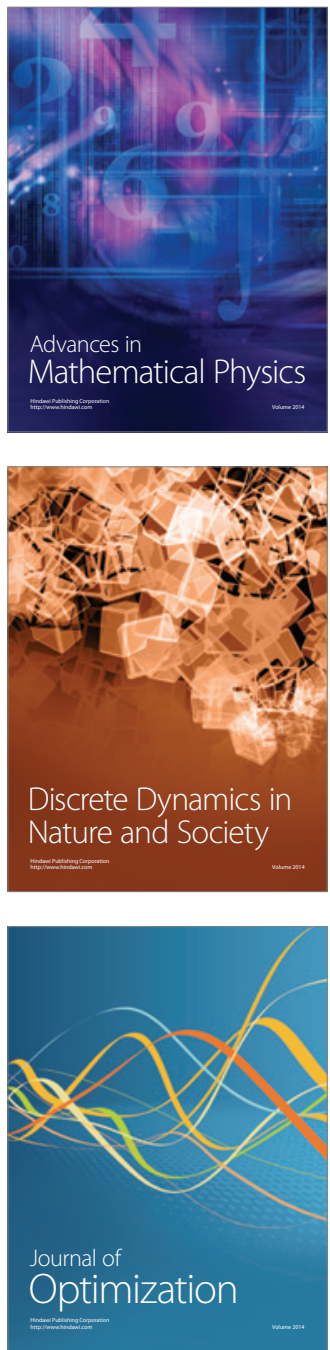